\title{
Nitric oxide and Endocan serum levels in sepsis
}

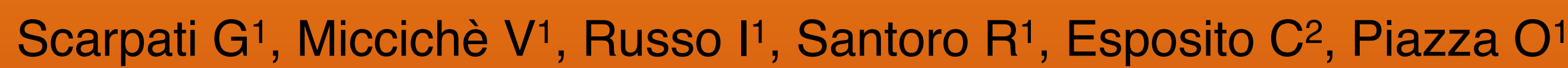

1 University of Salerno, Department of Medicine and Surgery, Baronissi (SA), Italy,

${ }^{2}$ Cardarelli Hospital, Dept of Anaesthesiology \& Intensive Care, Naples, Italy

Inflammation and endothelial activation are critical determinants of the host response and represent an explanation for the complex pathophysiology of sepsis.

Endocan is a novel sepsis marker; it is an endothelium derived soluble dermatan sulfate proteoglycan. It binds a wide range of bioactive molecules associated with cellular signaling and adhesion and thus regulating proliferation, differentiation, migration, and adhesion of different cell types in health and disease. An increase in tissue expression or serum level of endocan reflects endothelial activation and neovascularization which are prominent pathophysiological changes associated with inflammation.

Nitric oxide (NO) is the latest mediator to scrutinize with reference to the pathophysiology of sepsis; it has been shown to be particularly important as a mediator of vasoplegia, which is characteristic of the early, hyperdynamic phase of sepsis, and as a participant in myocardial depression.

This study aims to assess the diagnostic and prognostic value of endocan and nitric oxide in patients with SIRS and severe sepsis in a medical intensive care unit (ICU).

Materials and methods: We enrolled 13 severe sepsis patients and 7 SIRS patients (1). Endocan and NO serum levels were measured in patients with SIRS within 12 hours from diagnosis and in sepsis patients in the first, second and fourth day after diagnosis. NO serum levels were quantified by Sievers NO Analizer. Endocan serum levels were quantified by ELISA.

References: 1."American College of Chest Physicians/ Society of Critical Care Medicine Consensus Conference: definitions for sepsis and organ failure and guidelines for the use of innovative therapies in sepsis".

Crit. Care Med. 20 (6): 864-74.

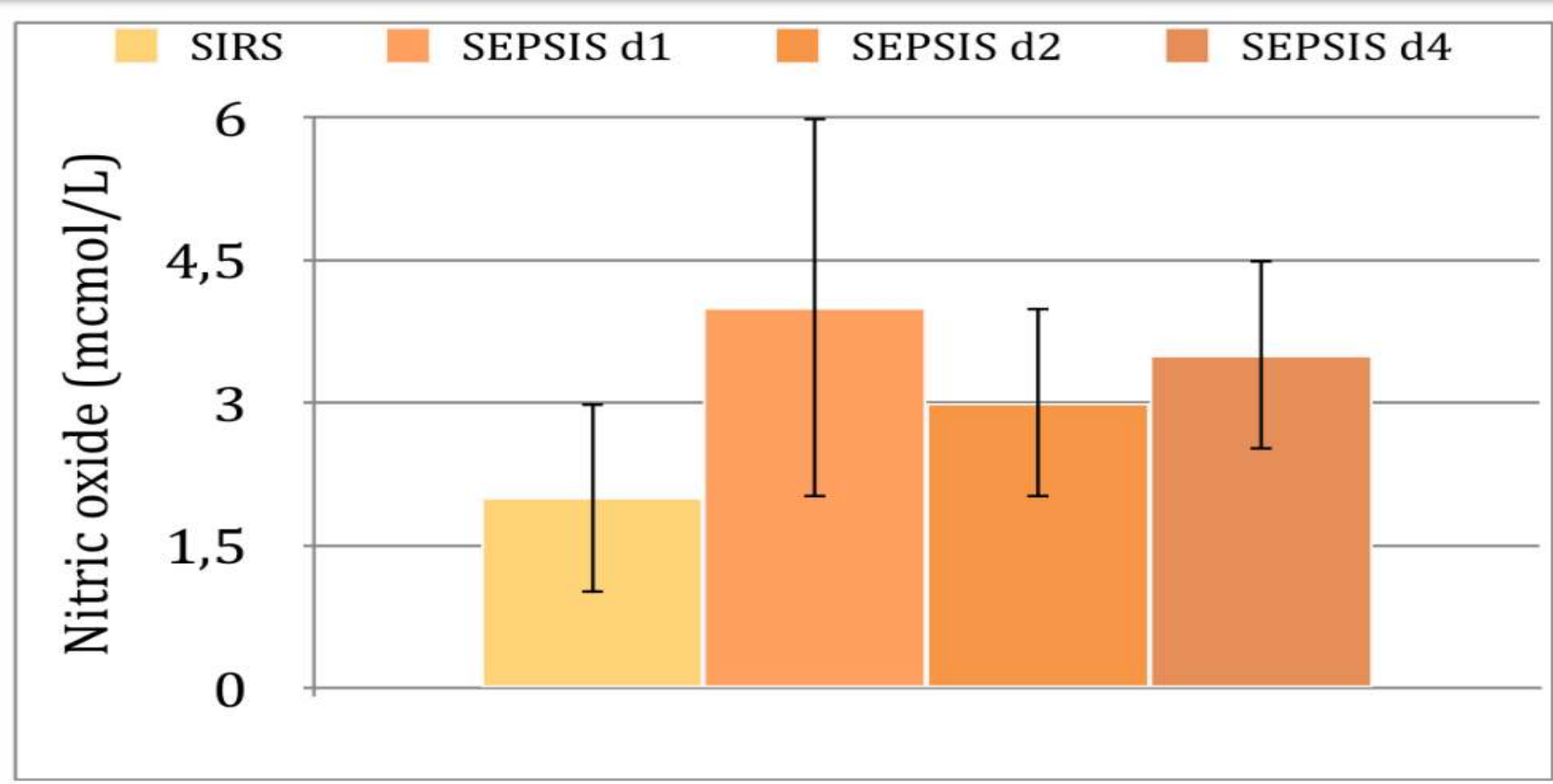

Figure 1: [Nitric Oxide serum levels in SIRS and sepsis.]

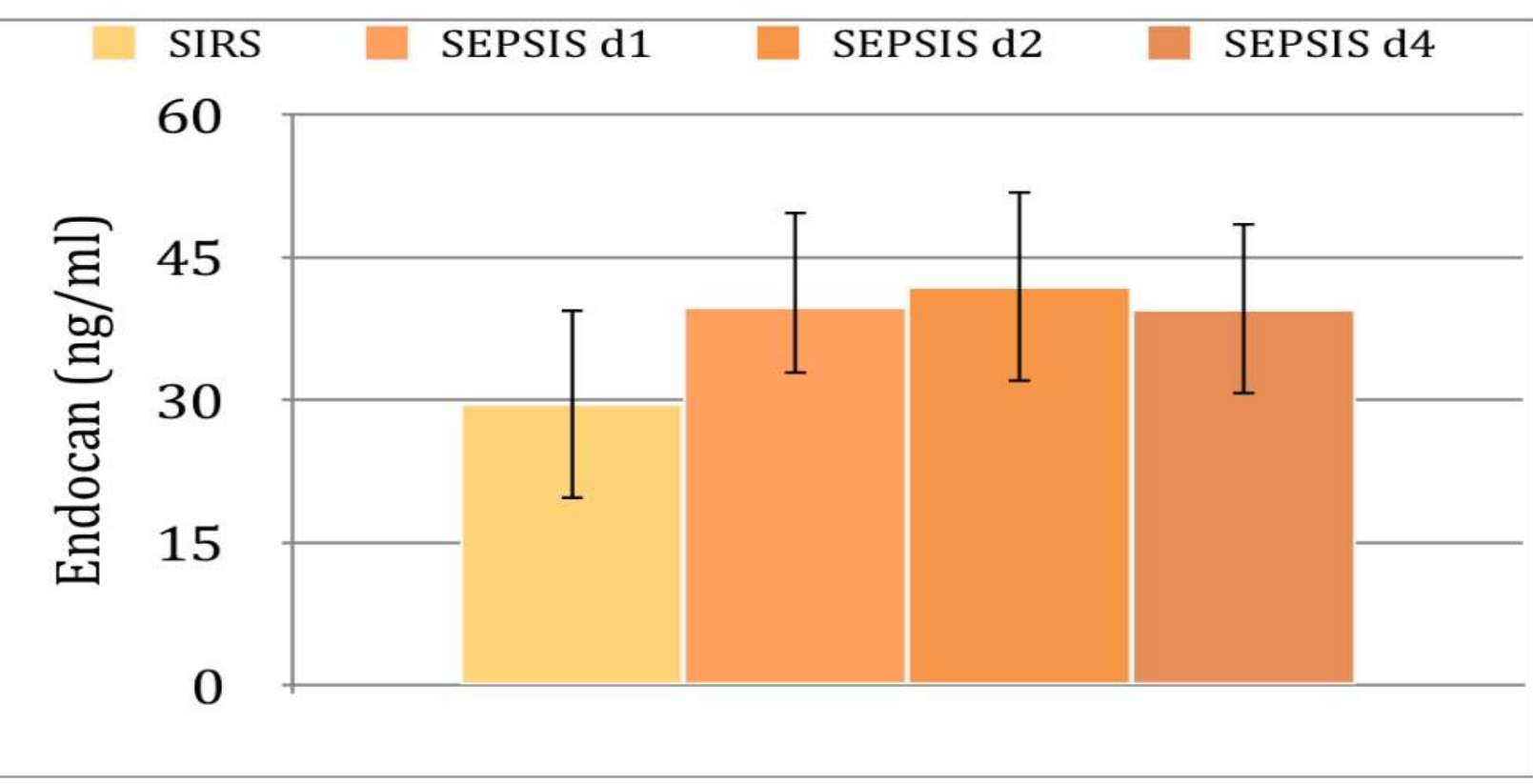

Figure 2: [Endocan serum levels in SIRS and sepsis]

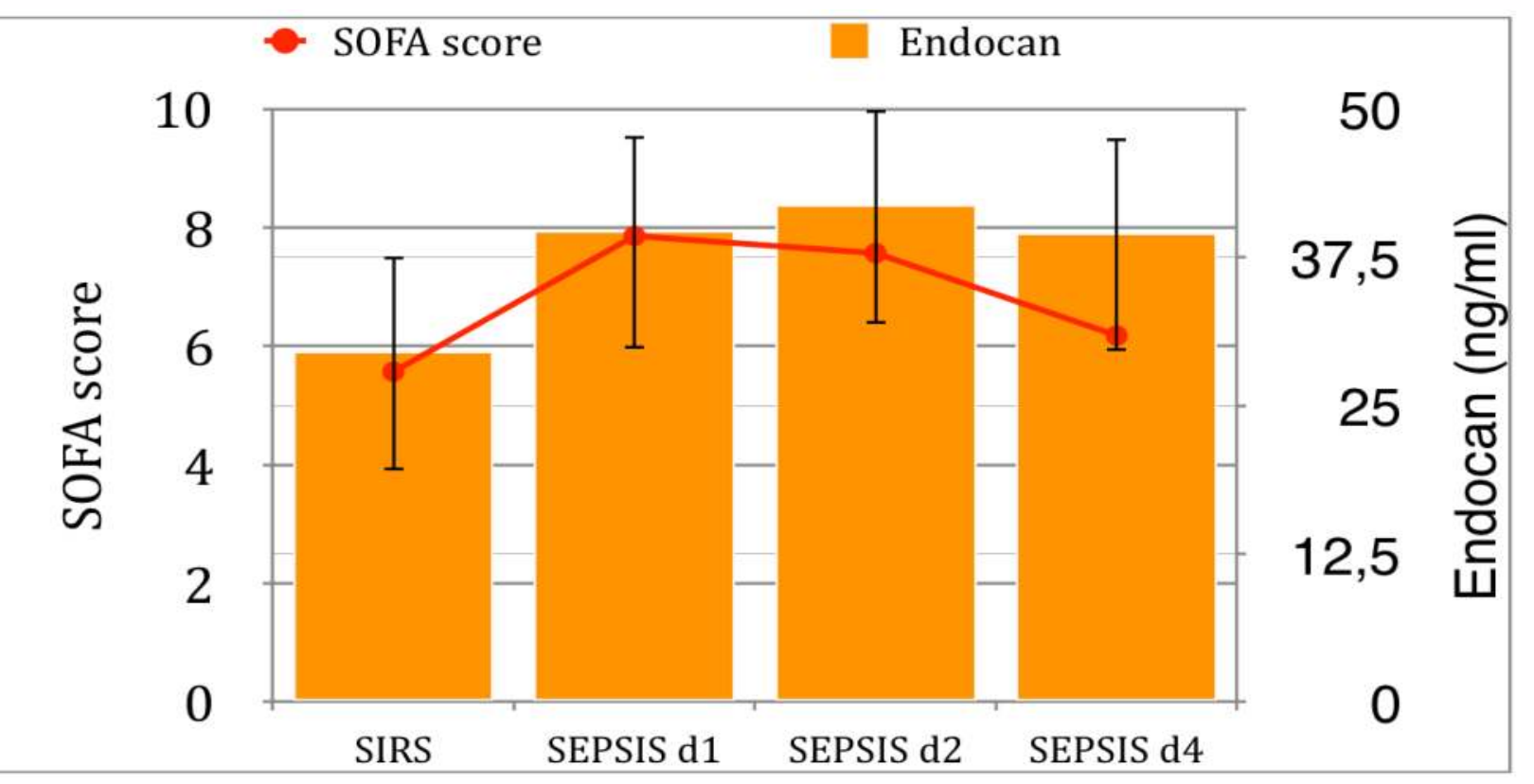

Figure 3: [Endocan serum levels and SOFA score in SIRS and sepsis]

Results: NO and endocan serum levels are significantly higher in sepsis patients than in SIRS ones. The values of $\mathrm{NO}$ and endocan are increased in patients with the highest values of SOFA (fig.3).

Conclusions: Serum endocan and NO levels in sepsis are higher than in SIRS. However, further prospective studies concerning the causative relationship between endocan and NO release are needed. 